\title{
Temperamento em bubalinos: testes de mensuração
}

\author{
Temperament in buffalos: mensurament tests
}

\author{
Sabina Secchin Scárdua ${ }^{{ }^{*}}$ Rosemary Bastos $^{\mathrm{I}}$ Carlos Ramon Ruiz Miranda ${ }^{\mathrm{II}}$
}

\section{RESUMO}

O objetivo neste trabalho foi avaliar a aplicabilidade, para búfalos (Bubalus bubalis), de testes de avaliação de temperamento, utilizados para bovinos. Foram avaliados os testes de docilidade, teste de reação a objetos novos, de tronco e de isolamento social em 12 bezerros e suas 12 mães, separadamente. Tanto os bezerros como suas mães responderam a todos os testes com vários comportamentos. Para os bezerros e as mães, os testes que promoveram maior número de comportamentos foram o de tronco $e$ de reação a objetos novos. Houve diferenças individuais (coeficiente de variação de $32-51 \%$ ) nos resultados de todos os testes tanto nos bezerros como nas mães. Os resultados individuais, em testes de isolamento social e de reação a objetos novos apresentaram correlação positiva tanto com as mães ( $r_{s}=$ $0,76, P<0,01)$ quanto com os bezerros $\left(r_{s}=0,66, P<0,01\right)$. Baseados em sensibilidade, relevância e aplicabilidade, o teste de isolamento social e o teste de tronco podem ser sugeridos para avaliarem o temperamento de bubalinos.

Palavras-chave: búfalos, manejo, produção animal, Bubalus bubalis.

\section{ABSTRACT}

The objective of this study was to assess the applicability of temperament tests commonly used in bovines for water buffaloes (Bubalus bubalis). The tests for docility, reaction to a novel object, squeeze cage and social isolation were applied individually with 12 calves and their 12 mothers. Both calves and their mothers responded to all tests with a variety of behaviors. The tests that elicited the larger number of behaviors were the squeeze cage and the novel object. There were large individual differences (coefficients of variation of $32-51 \%)$ in the scores for all tests for calves and adults. The individual scores for the tests of isolation and novel object were significantly correlated for calves $\left(r_{s}=0.66, P<0.01\right)$ and adults $\left(r_{s}=0.76, P<0.01\right)$. Using the criteria of sensitivity (range of individual differences), responsiveness and applicability, the squeeze cage test and the isolation test seem well suited for water buffaloes.

Key words: buffaloes, handling, animal production, Bubalus bubalis.

\section{INTRODUÇÃO}

Temperamento é um conceito antigo, usado para estabelecer um elo entre comportamento e constituição do indivíduo (ROTHBART \& DERRYBARRY, 1981). O desenvolvimento de padrões para a seleção de animais de temperamento apropriado, aliado aos estudos de métodos adequados de manejo, deve ser realizado para minimizar problemas e conseqüências negativas para os tratadores e os animais (LE NEINDRE et al., 1996). Para a produção animal, além da importância do estudo do temperamento no manejo, estudos revelam importância significativa do ponto de vista econômico, associando o temperamento à qualidade da carne (VOISINET et al., 1997), mortalidade de cordeiros (MARTIN et al., 2004) e fertilidade de machos (BURROW, 2001).

Para a mensuração do temperamento de um animal, são utilizados os chamados testes de

'Laboratório de Reprodução e Melhoramento Genético Animal, Universidade Estadual do Norte Fluminense (UENF). Av. Alberto Lamego, 2000, sala 26, 28013-600, Campos dos Goytacazes, RJ, Brasil. E-mail: sabina.scardua@bol.com.br.*Autor para correspondência.

"Laboratório de Ciências Ambientais, UENF, Campos dos Goytacazes, RJ, Brasil 
temperamento. O delineamento do teste, sua duração e os comportamentos avaliados variam entre diferentes abordagens experimentais. JONES \& GOSLING (2005) chamaram a atenção para a falta de padronização nos termos utilizados para descrever o temperamento em cães. Nas espécies de produção, principalmente bovinos, há também falta de padronização e definição dos escores utilizados (KILGOUR et al., 2006; SILVEIRA et al., 2006).

Um bom teste de temperamento será relevante seguro e discriminatório. O teste apresentará estímulo relevante para o animal, ou seja, o animal mostrará alguma reação, para que ser observada e avaliada. O teste deve ser seguro para o animal, para o observador e para o humano que é usado como estímulo, quando houver. O teste será sensível ou discriminatório, ou seja, é capaz de detectar diferenças entre indivíduos. Terá simples aplicação para assim ser utilizado nos vários tipos de criação e por pessoas diferentes. Será de rápida execução, para minimizar a possibilidade de ocorrência de fatores externos ao teste que influenciariam a resposta, barulho por exemplo (GRIGNARD et al., 2001).

Com o crescimento da bubalinocultura no Brasil, são imprescindíveis estudos do temperamento dos búfalos, bem como a avaliação dos métodos de mensuração dessa variável, visando ao bom desempenho do processo de seleção desses animais. O objetivo neste trabalho foi de avaliar testes de temperamento, comumente utilizados para bovinos, para a espécie bubalina.

\section{MATERIAL E MÉTODOS}

Foram utilizados 24 bubalinos mestiços murrah e jafarabadi, sendo 12 bezerros (idade entre três e cinco meses) e suas respectivas mães. O rebanho pertence ao Sítio Cruzeiro, propriedade particular no Município de Presidente Kennedy- ES. O rebanho é mantido em regime extensivo para fins de corte, com pastagem natural. Os animais passaram por um período de adaptação de dois meses à presença do observador e aos procedimentos de espera em curral e passagem pelo tronco de contenção.

A aplicação dos testes iniciou sempre pela manhã, estendendo-se até às $14 \mathrm{~h}$. Foram utilizados quatro testes para mensuração do temperamento: o teste de tronco (VOISINET et al., 1997; GRIGNARD et al., 2001); o teste de isolamento social (LANSADE et al., 2004); o teste de docilidade (GRIGNARD et al., 2001) e o teste de reação a objetos novos (RUIZ-MIRANDA \& CALLARD, 1992). Foi seguido um delineamento inteiramente casualizado em arranjo fatorial. Cada animal foi submetido a um teste por dia e a ordem dos testes foi distribuída ao acaso para cada animal. Os testes de isolamento, docilidade e reação ao objeto foram realizados na "seringa” (parte do curral que afunila o caminho para o tronco), medindo, aproximadamente, 8 metros de comprimento por cinco metros de largura e adjacente ao tronco e currais. As laterais da área do teste foram forradas com lona preta, o que impedia a visualização dos outros animais do mesmo rebanho, no curral ao lado. Todos os quatro testes tiveram duração de dez minutos e foram registrados, utilizandose câmera de vídeo, e o observador, operando a câmera, manteve-se visível ao animal apenas no teste do tronco, sendo o observador conhecido para o animal.

No teste de tronco, o animal foi encaminhado ao tronco de contenção e mantido preso por dois minutos sem a visualização de humanos ou indivíduos da mesma espécie, em seguida, foi fechada a lateral do tronco, restringindo ainda mais o movimento do animal, por mais cinco minutos e, por último, um humano que não pertencia ao convívio do animal foi posicionado a sua frente e permanecia imóvel por três minutos. No teste de isolamento social, o animal foi encaminhado até o local do teste e deixado lá por 10 minutos, sem estímulo adicional, além do isolamento físico e visual.

No teste de docilidade, o animal foi encaminhado ao local do teste e deixado com livre movimentação na seringa, depois de decorridos cerca de quatro minutos da permanência do animal na área coberta por lona, um humano, estranho ao animal, posicionava-se dentro da seringa, na entrada oposta à do animal, e se mantinha parado, ereto diante do animal por três minutos. Em seguida, o humano movimentava os braços lentamente e depois mais acelerado por cerca de um minuto, finalizando o teste.

No teste de reação a objetos novos, o animal foi encaminhado para a seringa, sendo que, no centro dessa, já se encontrava um objeto desconhecido para o animal. Foram utilizados cones de sinalização de trânsito, nas cores vermelha e branca, amarela e preta, medindo, aproximadamente, $80 \mathrm{~cm}$ de comprimento por $60 \mathrm{~cm}$ de base; cadeira de praia colorida; cadeira tradicional de madeira; banco plástico branco e sombrinha estampada em tons escuros. Os objetos, seguindo o mesmo padrão de tamanho e imobilidade e variando quanto à cor e forma, foram distribuídos ao acaso para os animais. Cada animal recebeu apenas um dos objetos.

Os dados registrados foram analisados, utilizando-se um vídeocassete, um cronômetro e uma planilha. Para cada teste, foram escolhidas as variáveis comportamentais a serem registradas (Tabela 1). A 
Tabela 1 - Variáveis comportamentais utilizadas nos quatro testes de temperamento.

\begin{tabular}{|c|c|c|}
\hline Teste & & Variável \\
\hline \multirow{7}{*}{ Isolamento } & & Andar \\
\hline & Mães & Investigar \\
\hline & & Excreção \\
\hline & & \\
\hline & & Andar \\
\hline & Bezerros & Investigar \\
\hline & & Vocalização \\
\hline \multirow{15}{*}{ Tronco } & & Investigar \\
\hline & & Orientação lateral da cabeça \\
\hline & & Excreção \\
\hline & Mães & Movimentação \\
\hline & & Tentativa de escape \\
\hline & & Orientado ao humano \\
\hline & & Aproximação do humano \\
\hline & & Investigar \\
\hline & & Orientação lateral da cabeça \\
\hline & & Vocalização \\
\hline & & Excreção \\
\hline & Bezerros & Movimentação \\
\hline & & Tentativa de escape \\
\hline & & Orientado ao humano \\
\hline & & Aproximação do humano \\
\hline \multirow{11}{*}{ Docilidade } & & Andar \\
\hline & & Investigar \\
\hline & & Vocalização \\
\hline & Mães & Excreção \\
\hline & & Orientado ao humano \\
\hline & & Aproximação do humano \\
\hline & & Andar \\
\hline & & Investigar \\
\hline & Bezerros & Vocalização \\
\hline & & Orientado ao humano \\
\hline & & Aproximação do humano \\
\hline \multirow{14}{*}{$\begin{array}{l}\text { Reação ao } \\
\text { objeto }\end{array}$} & \multirow{8}{*}{ Mães } & Andar \\
\hline & & Investigar \\
\hline & & Excreção \\
\hline & & Vocalização \\
\hline & & Adjacente ao objeto \\
\hline & & Orientado ao objeto \\
\hline & & Aproximação do objeto \\
\hline & & Interação com o objeto \\
\hline & \multirow{6}{*}{ Bezerros } & Andar \\
\hline & & Investigar \\
\hline & & Adjacente ao objeto \\
\hline & & Orientado ao objeto \\
\hline & & Aproximação do objeto \\
\hline & & Interação com o objeto \\
\hline
\end{tabular}

escolha foi baseada em ocorrência (ocorrência mínima em 1/3 dos animais) e testes preliminares de variância e correlação entre variáveis dentro do teste, em que variáveis, apresentando baixa variância e/ou alta correlação com outras variáveis de mesma categoria, foram descartadas. As variáveis comportamentais utilizadas nos diferentes testes foram agrupadas nas categorias reatividade geral, docilidade e exploração.

Nas planilhas individuais para cada teste, foram registradas as variáveis comportamentais, levando-se em consideração, para cada uma, o tempo de duração do comportamento, ou seja, tempo total durante o teste em que o animal executou aquele comportamento; a latência para execução do comportamento, ou seja, quanto tempo se passava desde o início do teste até a execução do comportamento pela primeira vez e número de ocorrência do comportamento, ou seja, quantas vezes, em um teste, o animal executava o mesmo comportamento.

Para duração e latência (em segundos) e número de ocorrência de cada comportamento em cada teste, foi feito um ranking dos doze indivíduos (mães foram analisadas separadamente dos bezerros) para determinação dos escores. O indivíduo correspondente ao primeiro lugar no ranking (maior valor) recebeu o escore máximo (12) e o indivíduo correspondente ao último lugar (menor valor) recebeu o escore mínimo (1 - para menor valor numérico e 0 - para ausência do comportamento). A latência recebeu escore invertido, ou seja, quanto menor a latência maior o escore. O escore, para cada comportamento, foi obtido somando-se os escores de duração, latência e ocorrência. O escore total do indivíduo, no teste, foi feito somando-se os escores para todos os comportamentos do teste e após a soma individual, os animais foram ordenados de 1 a 12 .

A avaliação de cada teste de temperamento foi realizada através da verificação de três aspectos: a sensibilidade, a relevância e aplicabilidade, de acordo com GRIGNARD et al. (2001). A sensibilidade do teste, ou seja, a capacidade de detectar diferenças entre indivíduos foi constatada através do coeficiente de variação do teste e através dos diferentes comportamentos mostrados durante o teste. A relevância do estímulo oferecido ao animal para expressar seu comportamento foi constatada através do número de variáveis comportamentais apresentadas pelos animais. A aplicabilidade do teste, ou seja, a facilidade de aplicação na propriedade, foi constatada subjetivamente, levando-se em consideração a logística de aplicação e principalmente o risco para as pessoas envolvidas. Os testes foram avaliados separadamente para fêmeas adultas e bezerros.

Foi feita análise de variância, considerando os fatores idade e teste e sua interação, além de testes de comparação de médias Tukey, adotando-se a 5\% de probabilidade, através do programa SAS. Foi verificada a associação entre testes, determinando-se o 
coeficiente de correlação de Spearman. Dessa forma, testes com escores individuais correlacionados significativamente seriam considerados redundantes. Foi utilizado o programa SPSS versão 6.0, adotando-se 5\% de probabilidade.

\section{RESULTADOS}

Nos quatro testes, houve relevância e sensibilidade. Os quatro testes apresentam relevância para a espécie bubalina, de acordo com o número final de variáveis comportamentais apresentadas, ou seja, após eliminação de comportamentos correlacionados através de análise preliminar (Isolamento social mães e bezerros $=3$; tronco mães $=7$ e bezerros $=8$; docilidade mães $=6$ e bezerros $=5$; reação a objetos novos mães = 8 e bezerros $=6$ ). No teste de isolamento social, apresentaram-se duas varáveis comportamentais em comum para bezerros e mães, enquanto a terceira diferiu, sendo excreção para mães e vocalização para bezerros. No teste de tronco, sete variáveis foram comuns para mães e bezerros, diferindo em vocalização para bezerros. No teste de docilidade, as mães apresentaram a variável excreção a mais que os bezerros e, no teste de reação a objetos novos, as mães apresentaram vocalização e excreção como variáveis adicionais, quando comparadas aos bezerros (Tabela 1).

A sensibilidade dos testes está apresentada na tabela 2, através de seus coeficientes de variação. Indivíduos mostraram diferenças individuais nas variáveis quantitativas que exibiram em comum, mas também houve diferenças qualitativas nos comportamentos exibidos. Dentro de um teste, por exemplo, alguns animais exibiram apenas o comportamento investigar, enquanto outros exibiram andar e excreção em vez de investigar. O escore dos animais, de uma forma geral, não se manteve ao longo dos quatro testes aplicados (Figuras 1 e 2). Os testes de isolamento social e de reação a objetos novos, porém, apresentaram correlação positiva tanto com as mães $\left(\mathrm{r}_{\mathrm{s}}=0,76, \mathrm{P}<0,01\right)$ quanto com os bezerros $\left(\mathrm{r}_{\mathrm{s}}=\right.$ $0,66, \mathrm{P}<0,01)$.

A análise de variância não revelou efeito significativo para a idade dos animais $(\mathrm{F}=0,06, \mathrm{P}<0,8)$ e interação dos fatores idade e teste $(\mathrm{F}=0,8, \mathrm{P}<0,49)$. No entanto, houve efeito significativo dos testes $(\mathrm{F}=$ $2,86, \mathrm{P}<0,04)$. As médias dos testes de isolamento social e de tronco diferiram, significativamente, entre si, pelo teste de Tukey, em nível de $5 \%$ de probabilidade. O teste de docilidade não diferiu significativamente dos testes de isolamento social e reação a objetos novos, enquanto o teste de reação a objetos novos também não diferiu do teste de tronco.

Quanto à aplicabilidade, no teste de reação a objetos novos, de docilidade e de isolamento social, necessita-se de preparação do local antes do início do teste (colocação de lona), enquanto, no teste de reação a objetos novos, era um agravante logístico: um objeto inteiramente novo para o animal. No teste de docilidade, apresenta-se risco para o humano, utilizado como estímulo.

\section{DISCUSSÃO}

Os quatro testes utilizados mostraram-se relevantes para a espécie bubalina, ou seja, os animais responderam, através dos comportamentos apresentados, aos testes aplicados. A relevância de um teste de temperamento é um fator fundamental, como preconizado por GRIGNARD et al. (2001), que destacaram que um bom teste deve ser relevante, discriminatório, de rápida execução e seguro.

Os testes utilizados apresentaram sensibilidade para a espécie bubalina. Pelos testes detectaram-se diferenças quantitativas entre os indivíduos, indicadas pelo coeficiente de variação, que se manteve acima de 30\%, valor máximo, considerado comum para resposta animal, de acordo com SAMPAIO (2007). Também houve diferença qualitativa nas respostas, entre bezerros e mães e dentro do grupo de bezerros e mães, o que sugere que os testes permitem aos animais expressarem seu temperamento de forma individual, já que os indivíduos podem apresentar comportamentos diferentes, provocados pelo mesmo estímulo externo.

A análise de variância não revelou efeito significativo do fator idade, o que indica que, apesar de os bezerros e mães apresentarem variações

Tabela 2 - Coeficientes de Variação (\%) dos diferentes testes utilizados para fêmeas adultas e bezerros.

\begin{tabular}{lcccc}
\hline & & \multicolumn{2}{c}{ Coeficientes de Variação } \\
\hline Animais & Docilidade & Tronco & Isolamento & Objeto \\
Fêmeas adultas & $51,6 \%$ & $32,1 \%$ & $44,8 \%$ & $37,4 \%$ \\
Bezerros & $37,0 \%$ & $33,4 \%$ & $31,9 \%$ & $40,7 \%$ \\
Média & $44,3 \%$ & $32,7 \%$ & $38,3 \%$ & $39 \%$ \\
\hline
\end{tabular}

Ciência Rural, v.39, n.2, mar-abr, 2009. 


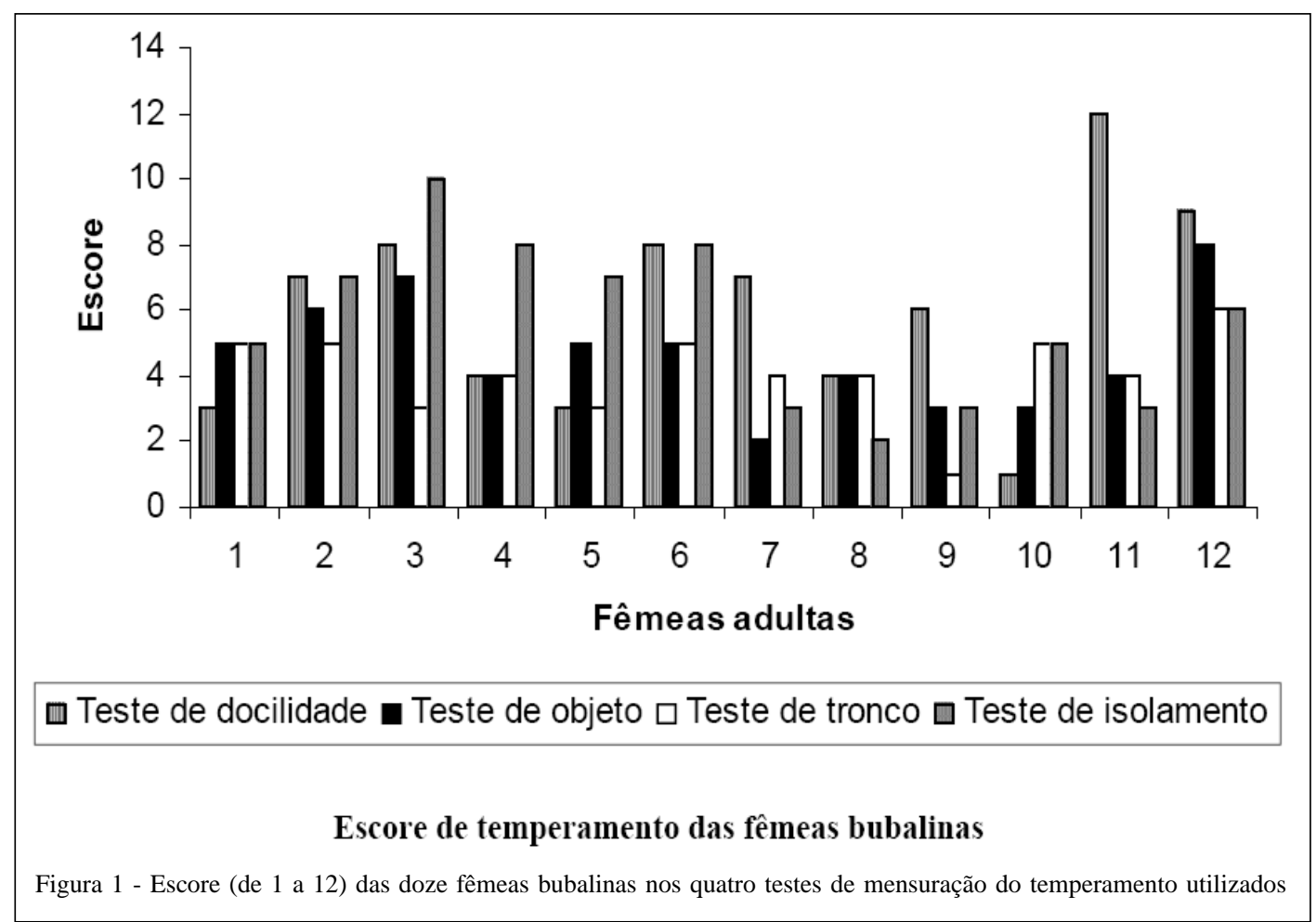

quantitativas e qualitativas em suas respostas aos diferentes testes, bezerros a partir de três meses de idade não precisam de testes específicos para eles. Portanto os bubalinos jovens podem ser avaliados pelos mesmos testes utilizados para adultos.

O teste de comparação de médias destaca os testes de tronco e isolamento social como diferentes entre si, o que reforça a escolha desses dois testes para uso em bubalinos. GRANDIN (1998) cita o teste de tronco como melhor avaliador dos fatores genéticos do temperamento, enquanto o teste de isolamento social inclui os fatores do temperamento associados ao desenvolvimento do animal, aprendizado e experiência. O escore, nos dois testes, pode então ser ponderado para classificar o animal em reatividade baixa, média ou alta, mas principalmente, a apresentação de dois testes ao indivíduo é importante para permitir respostas de caráter definitivo ou eliminatório, como por exemplo, tentativa de agressão ao humano presente no teste do tronco, ou tentativa de escape da seringa, no teste de isolamento. Esses comportamentos não foram apresentados no presente trabalho, possivelmente, por tratar-se de um rebanho pequeno.

$\mathrm{O}$ escore dos animais não se manteve ao longo dos quatro testes utilizados. A inconsistência dos escores atesta que o temperamento é complexo e que o escore de um teste não prediz o escore em outro, assim como foi encontrado por LANSADE et al. (2004), trabalhando com eqüinos. Esses autores recomendam a utilização de mais de um teste para avaliar o temperamento de um animal. Há testes correlacionados, que poderiam ser considerados redundantes. $\mathrm{O}$ teste de isolamento social e reação a objetos novos foram correlacionados. Trata-se de testes parecidos, pois se baseiam em impor ao animal uma situação estranha, mas sem perigo. Uma correlação significativa entre o teste de tronco e docilidade em bovinos foi demonstrada por GRIGNARD et al. (2001), o que não foi observado para búfalos neste trabalho. SEAMAN et al. (2002) avaliaram os testes de isolamento social, docilidade e reação a objetos novos para eqüinos e encontraram correlação entre os dois últimos. Esses autores sugeriram o teste de isolamento social como o melhor teste para a espécie eqüina, baseado na aplicabilidade e consistência através do tempo.

Quanto à presença de humano cuja importância foi salientada por GRIGNARD et al. (2001), o teste de tronco também apresenta o humano como estímulo, o que supera a necessidade de trabalhar com o teste de docilidade que pode ser considerado de alto risco, quando se trabalha com animais do porte da espécie bubalina. Para a espécie bovina, KILGOUR et al. (2006) sugeriram como melhores testes, o de tronco e o de isolamento social. No presente trabalho, 


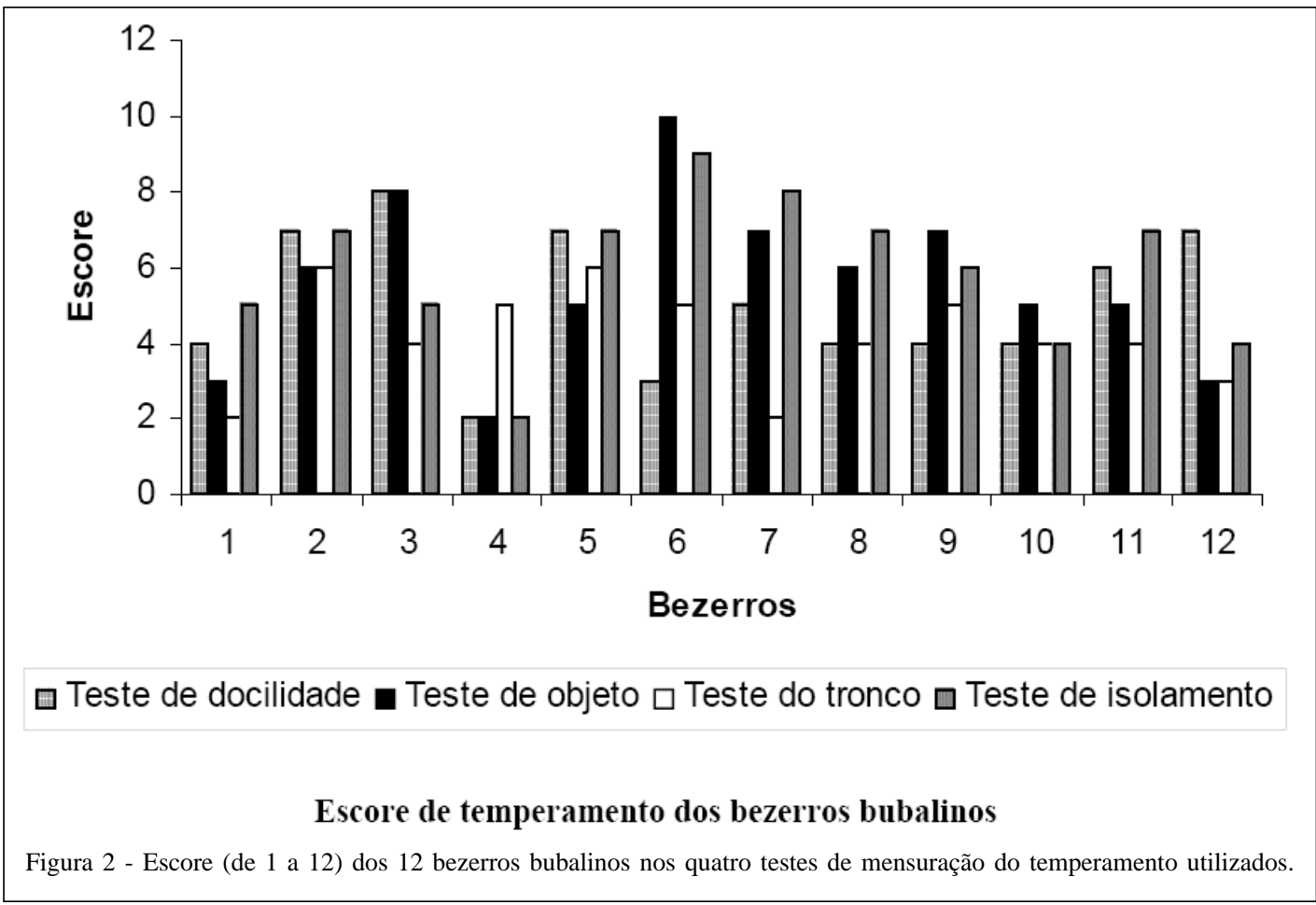

com relação à aplicabilidade do teste, os testes de tronco e de isolamento social se mostraram os melhores, já que o de reação a objetos novos necessita de um objeto inteiramente novo para o animal e apresentou correlação positiva com o teste de isolamento social, enquanto o teste de docilidade não é seguro.

De acordo com SEAMAN et al. (2002), nenhum dos diferentes tipos de testes pode ser usado sozinho para executar um acesso acurado do temperamento do animal nem para predizer respostas em outros tipos de testes. Procurou-se então, no presente trabalho, escolher dois dos quatro testes para serem utilizados em bubalinos. Portanto, ponderandose os resultados, pode-se sugerir o teste de tronco e de isolamento social como bons avaliadores do temperamento bubalino.

\section{CONCLUSÃO}

Baseado em sensibilidade, relevância e aplicabilidade, o teste de isolamento e o teste de tronco podem ser sugeridos para avaliação do temperamento de indivíduos, tanto jovens como adultos, da espécie Bubalus bubalis.

\section{AGRADECIMENTOS}

Os autores agradecem ao proprietário da fazenda, Sr. Elmo Tarso Santana e ao funcionário da fazenda, Sr. Antônio
Carlos Moura, pela permissão e colaboração para realização do experimento. Esta pesquisa teve suporte financeiro da FAPERJ (Processo n ${ }^{\circ}$ E26/170.645/2003).

\section{COMITÊ DE ÉTICA E BIOSSEGURANÇA}

Este projeto foi avaliado e aprovado pelo Comitê de Ética da Universidade Estadual do Norte Fluminense - UENF.

\section{REFERÊNCIAS}

BURROW, H.M. Variances and covariances between productive and adaptive traits and temperament in a composite breed of tropical beef cattle. Livestock Production Science, v.70, p.213-233, 2001.

GRANDIN, T. Review: reducing handling stress improves both productivity and welfare. Professional Animal Scientist, v.14, n.1, p.1-15, 1998

GRIGNARD, L. et al. Do beef cattle react consistently to different handling situations? Applied Animal Behaviour Science, v.71, p.263-276, 2001.

JONES, A.C.; GOSLING, S.D. Temperament and personality in dogs (Canis familiaris): a review and evaluation of past reserch. Applied Animal Behavioural Science, v.95, p.1-53, 2005.

KILGOUR, R.J. et al. Individual differences in reacton of beef cattle to situations invilving social isolation, close proximity of humans, restraint and novelty. Applied Animal Behavioural Science, v.99, p.21-40, 2006.

Ciência Rural, v.39, n.2, mar-abr, 2009. 
LANSADE, L. et al. Effects of handling at weaning on manageability and reactivity of foals. Applied Animal Behaviour Science, v.87, p.131-149, 2004.

LE NEINDRE, P. et al. Handling of extensively kept animals. Applied Animal Behaviour Science, v.49, p.73-81, 1996.

MARTIN, G.B. et al. Natural methods for increasing reproductive efficiency in small ruminants. Animal Reproduction Science, v.82, p.231-246, 2004.

ROTHBART, M.K.; DERRYBARRY, D. Development of individual differences in temperament. In: LAMB, M.E.; BROWN, A.L. Advances in developmental psychology. Hillsdale: Lawrence Erlbaum Associates Publishers, 1981. p.3785.

RUIZ-MIRANDA, C.R.; CALLARD, M. Effects of the presence of the mother on responses of domestic goat kids (Capra hircus) to novel inanimate objects and humans.
Applied Animal Behaviour Science, v.33, p.277285,1992.

SAMPAIO, I.B.M. Estatística aplicada à experimentação animal. Belo Horizonte: Fundação de Estudo e Pesquisa em Medicina Veterinária e Zootecnia, 2007. 264p.

SEAMAN, S.C. et al. How reliable is temperament assessment in the domestic horse (Equus caballus)? Applied Animal Behaviour Science, v.78, p.175-191, 2002.

SILVEIRA, I.D.B. et al. Comportamento de bovinos de corte em pista de remate. Ciência Rural, Santa Maria, v.36, n.5, p.1529-1533, 2006.

VOISINET, B.D. et al. Bos indicus-cross feedlot cattle with excitable temperaments have tougher meat and a higher incidence of borderline dark cutter. Meat Science, v. 46, n.4, p.367-377, 1997. 